\title{
Temperature-dependent effective third-order interatomic force constants from first principles
}

\author{
Olle Hellman and Igor Abrikosov
}

\section{Linköping University Post Print}

\section{Tweet}

N.B.: When citing this work, cite the original article.

Original Publication:

Olle Hellman and Igor Abrikosov, Temperature-dependent effective third-order interatomic force constants from first principles, 2013, Physical Review B. Condensed Matter and Materials Physics, (88), 14.

http://dx.doi.org/10.1103/PhysRevB.88.144301

Copyright: American Physical Society http://www.aps.org/ 


\title{
Temperature-dependent effective third-order interatomic force constants from first principles
}

\author{
Olle Hellman and I. A. Abrikosov \\ Department of Physics, Chemistry and Biology (IFM), Linköping University, SE-581 83, Linköping, Sweden
}

(Received 11 July 2013; published 1 October 2013)

\begin{abstract}
The temperature-dependent effective potential (TDEP) method is generalized beyond pair interactions. The second- and third-order force constants are determined consistently from ab initio molecular dynamics simulations at finite temperature. The reliability of the approach is demonstrated by calculations of the mode Grüneisen parameters for $\mathrm{Si}$. We show that the extension of TDEP to a higher order allows for an efficient calculation of the phonon life time, in $\mathrm{Si}$ as well as in $\epsilon$-FeSi; a system that exhibits anomalous softening with temperature.
\end{abstract}

DOI: 10.1103/PhysRevB.88.144301

PACS number(s): 63.20.-e, 63.20.dk, 63.20.K-

\section{INTRODUCTION}

Thermodynamic properties of materials are often discussed in terms of the quasiharmonic approximation. ${ }^{1,2}$ This theory has a solid foundation, but it is not without limitations. Any property that relies on phonon lifetimes and scattering rates, such as thermal conductivity, is unavailable. For those properties one needs terms higher than second order in the Taylor expansion of the crystal potential energy surface. If the higher-order terms are known, there is extensive theory developed for the properties that can be extracted. ${ }^{3}$ The difficulties lie in determining these parameters.

Density functional theory gives one access to the potential energy surface. Perturbation theory and the $2 n+1$ theorem or direct supercell approaches can be used to determine materials' force constants. ${ }^{4-6}$ In these formalisms, however, the potential energy surface is treated as constant with respect to temperature. We have previously shown that this is not the case. ${ }^{7,8}$ In those studies, using the temperature dependent effective potential (TDEP), we obtain the best possible second order Hamiltonian as a fit to the Born-Oppenheimer molecular dynamics potential energy surface at finite temperature. With this technique it is possible, for example, to accurately describe solid ${ }^{4} \mathrm{He}$, which is strongly anharmonic, with a second-order Hamiltonian. The effective potential gives accurate phonon dispersion relations and free energies. The aim of this paper is to extend the TDEP formalism to include higher-order terms, making the technique suitable for calculations of important materials properties such as phonon lifetimes.

\section{METHOD}

We start with a model crystal Hamiltonian

$$
\begin{aligned}
H= & U_{0}+\sum_{i} \frac{m \mathbf{p}_{i}^{2}}{2}+\frac{1}{2 !} \sum_{i j \alpha \beta} \Phi_{i j}^{\alpha \beta} u_{i}^{\alpha} u_{j}^{\beta} \\
& +\frac{1}{3 !} \sum_{i j k \alpha \beta \gamma} \Psi_{i j k}^{\alpha \beta \gamma} u_{i}^{\alpha} u_{j}^{\beta} u_{k}^{\gamma} .
\end{aligned}
$$

Here $U$ is the potential energy and $\bar{\Phi}_{i j}$ and $\overline{\bar{\Psi}}_{i j k}$ are the secondand third-order force constants. The displacement of atom $i$ from ideal positions is denoted $\vec{u}_{i}$, its momentum $\vec{p}_{i}$, and $\alpha \beta \gamma$ are Cartesian indices. Bold symbols indicate vectors and doubly overlined symbols matrices or tensors, respectively.

The basic idea of the generalized TDEP is to use BornOppeheimer molecular dynamics to accurately sample the potential energy surface at finite temperature. Then we fit the model Hamiltonian in Eq. (1) to this surface. This is done by comparing the forces of the model and the ab initio system at each time step and minimizing the difference.

With the vast number of values to be determined for the third-order force constants it is crucial for the generalization of the TDEP to higher-order terms to make use of the symmetry analysis. ${ }^{8}$ We begin by reiterating the symmetry relations the force constants obey, ${ }^{2}$ first the transposition symmetries

$$
\begin{gathered}
\Phi_{i j}^{\alpha \beta}=\Phi_{j i}^{\beta \alpha}, \\
\Psi_{i j k}^{\alpha \beta \gamma}=\Psi_{i k j}^{\alpha \gamma \beta}=\Psi_{j i k}^{\beta \alpha \gamma}=\Psi_{j k i}^{\beta \gamma \alpha}=\Psi_{k i j}^{\gamma \alpha \beta}=\Psi_{k j i}^{\gamma \beta \alpha} .
\end{gathered}
$$

Then, if two tensors are related by symmetry operation $S$ the components are related as follows:

$$
\begin{gathered}
\Phi_{i j}^{\alpha \beta}=\sum_{\mu \nu} \Phi_{k l}^{\mu \nu} S^{\mu \alpha} S^{\nu \beta}, \\
\Psi_{i j k}^{\alpha \beta \gamma}=\sum_{\mu \nu \xi} \Psi_{m n o}^{\mu \nu \xi} S^{\mu \alpha} S^{\nu \beta} S^{\xi \gamma} .
\end{gathered}
$$

Force constants also obey the acoustic sum rules

$$
\begin{gathered}
\sum_{j} \overline{\bar{\Phi}}_{i j}=0 \quad \forall i, \\
\sum_{k} \overline{\bar{\Psi}}_{i j k}=0 \quad \forall i, j .
\end{gathered}
$$

To apply symmetry relations (2) to (7), we set each tensor component to a symbolic variable, called $\theta_{k}$. The index $k$ runs from 1 to the total number of components in all tensors. We include all tensors within a cutoff radius $r_{c}$ (the maximum cutoff is determined by the simulation cell size). Using Eqs. (2) through (7) we figure out which tensor components that are equal, related to each other or 0 by symmetry. This drastically reduces the number of values that have to be determined. With the symmetry irreducible representation at hand, we express the forces in the model Hamiltonian

$$
F_{i}^{\alpha}=\sum_{j \beta} \Phi_{i j}^{\alpha \beta} u_{j}^{\beta}+\frac{1}{2} \sum_{j k \beta \gamma} \Psi_{i j k}^{\alpha \beta \gamma} u_{j}^{\beta} u_{k}^{\gamma} .
$$

Evaluating Eq. (8) analytically allows to express the forces as a function of the symmetry inequivalent components $\theta_{k}$ :

$$
F_{i}^{\alpha}=\sum_{k} \theta_{k} c_{k}^{i \alpha}(\mathbf{U}) .
$$

Here $c_{k}^{i \alpha}(\mathbf{U})$, the coefficient for each $\theta_{k}$ is a polynomial function of all displacements within $r_{c}$. The form of this function 
depends on the crystal at hand. For a given supercell, we can express Eq. (9) as a matrix equation

$$
\mathbf{F}_{M}=\Theta \overline{\overline{\mathbf{C}}}(\mathbf{U}) \text {. }
$$

The subscript $M$ denotes the forces from the model potential. The coefficent matrix $\overline{\overline{\mathbf{C}}}$ is a function of all the displacements in the supercell. $\Theta$ is a vector holding all the $\theta_{k}$. To obtain a solution for $\Theta$ we run Born-Oppenheimer molecular dynamics in the canonical ensemble at temperature $T$. From these simulations, we store displacements $\mathbf{u}$ and forces $\mathbf{F}_{M D}$ at each time step. Then, we seek the $\Phi$ that minimize the difference between the model system and the ab initio one

$$
\begin{aligned}
\min _{\Theta} \Delta \mathbf{F} & =\frac{1}{N_{t}} \sum_{t=1}^{N_{t}}\left|\mathbf{F}_{t}^{\mathrm{MD}}-\mathbf{F}_{t}^{\mathrm{H}}\right|^{2} \\
& =\frac{1}{N_{t}} \sum_{t=1}^{N_{t}}\left|\mathbf{F}_{t}^{\mathrm{MD}}-\overline{\overline{\mathbf{C}}}\left(\mathbf{U}_{t}^{\mathrm{MD}}\right) \Theta\right|^{2} \\
& =\frac{1}{N_{t}}\left\|\left(\begin{array}{c}
\mathbf{F}_{1}^{\mathrm{MD}} \\
\vdots \\
\mathbf{F}_{N_{t}}^{\mathrm{MD}}
\end{array}\right)-\left(\begin{array}{c}
\overline{\overline{\mathbf{C}}}\left(\mathbf{U}_{1}^{\mathrm{MD}}\right) \\
\vdots \\
\overline{\overline{\mathbf{C}}}\left(\mathbf{U}_{N_{t}}^{\mathrm{MD}}\right)
\end{array}\right) \Theta\right\|^{2} .
\end{aligned}
$$

Here $N_{t}$ is the number of time steps in the molecular dynamics, and subscript $t$ denotes the displacements and forces from time step $t$. A least-square solution

$$
\Theta=\left(\begin{array}{c}
\overline{\overline{\mathbf{C}}}\left(\mathbf{U}_{1}^{\mathrm{MD}}\right) \\
\vdots \\
\overline{\overline{\mathbf{C}}}\left(\mathbf{U}_{N_{t}}^{\mathrm{MD}}\right)
\end{array}\right)^{+}\left(\begin{array}{c}
\mathbf{F}_{1}^{\mathrm{MD}} \\
\vdots \\
\mathbf{F}_{N_{t}}^{\mathrm{MD}}
\end{array}\right)
$$

gives the $\Theta$ that minimizes these forces. Then, with a simple substitution back into $\Phi_{i j}$ and $\Psi_{i j k}$ we determine the quadratic and cubic force constants. Note that the second- and thirdorder force constants are extracted from the same set of displacements and forces, simultaneously.

\section{NUMERICAL RESULTS}

$\mathrm{Si}$ is a common model system where high-order terms are relevant, ${ }^{4,6,9,10}$ and to verify the quality of the force constants obtained in the TDEP formalism we employ Born-Oppenheimer molecular dynamics with the projectoraugmented wave (PAW) method as implemented in the code VASP. ${ }^{11-14}$ We use a 128-atom supercell. For the Brillouin zone integration we use the $\Gamma$-point and ran the simulations on a grid of temperatures and volumes in the canonical ensemble. Temperature was controlled using a Nosé thermostat. ${ }^{15}$ Exchangecorrelation effects were treated using the generalized gradient approximation with the Perdew-Burke-Ernzerhof ${ }^{16}$ functional. We use a plane wave cutoff of $250 \mathrm{eV}$. The simulations ran for about 100 ps with a time step of $1 \mathrm{fs}$. A subset of uncorrelated samples is then chosen. For each of the samples the electronic structure and total energies are recalculated using a $5 \times 5 \times 5$ $k$-point grid and a cutoff of $500 \mathrm{eV}$.

The first way of verifying if our force constants are correct is to calculate the potential energy according to Eq. (1) and compare to the ones from density functional theory (DFT). In Fig. 1 we show the difference in potential energy from

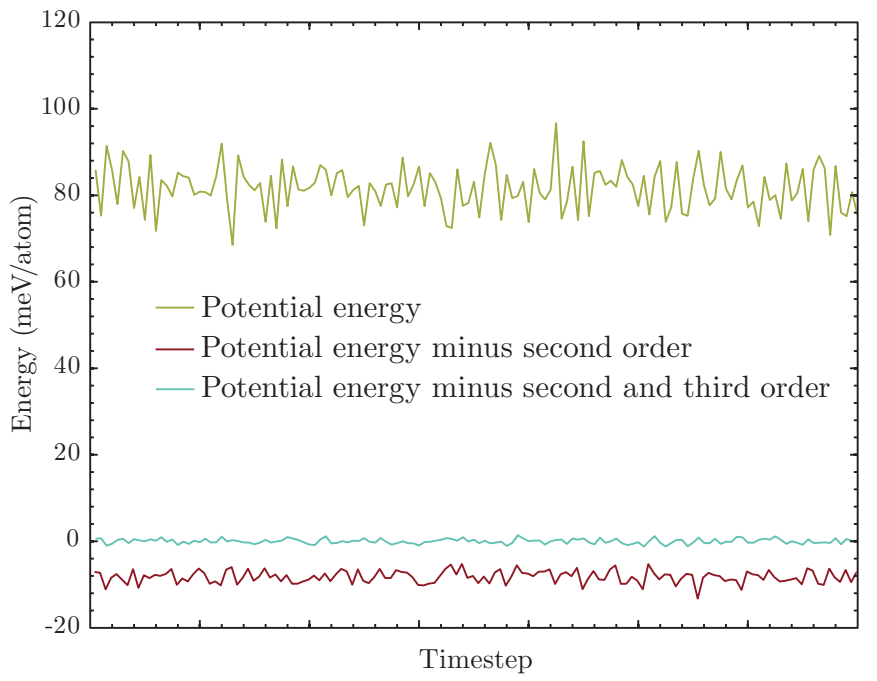

FIG. 1. (Color online) Figure showing the difference between the ab initio potential energies of $\mathrm{Si}$ and the potential energies from our model potential. The top line is the values from Born-Oppenheimer molecular dynamics. The bottom line is the ab initio energies with the second-order term in Eq. (1) subtracted, and the middle line is when both the second- and third-order energies are subtracted. Had the model potential been exact, the middle line would be perfectly straight. There are still some fluctuations, but they are on the order of $1 \mathrm{meV} /$ atom, and the accuracy of our potential is good. The secondorder potential includes up to fourth neighbors and the third-order up to second nearest neighbors.

DFT and the model Hamiltonian, and in Fig. 2 we show the convergence of this potential energy with respect to the number of time steps. These results confirm that the third-order force constants are accurately determined and represent the potential energy surface well.

The mode Grüneisen parameters are a measure how sensitive the vibrational frequencies are with respect to a

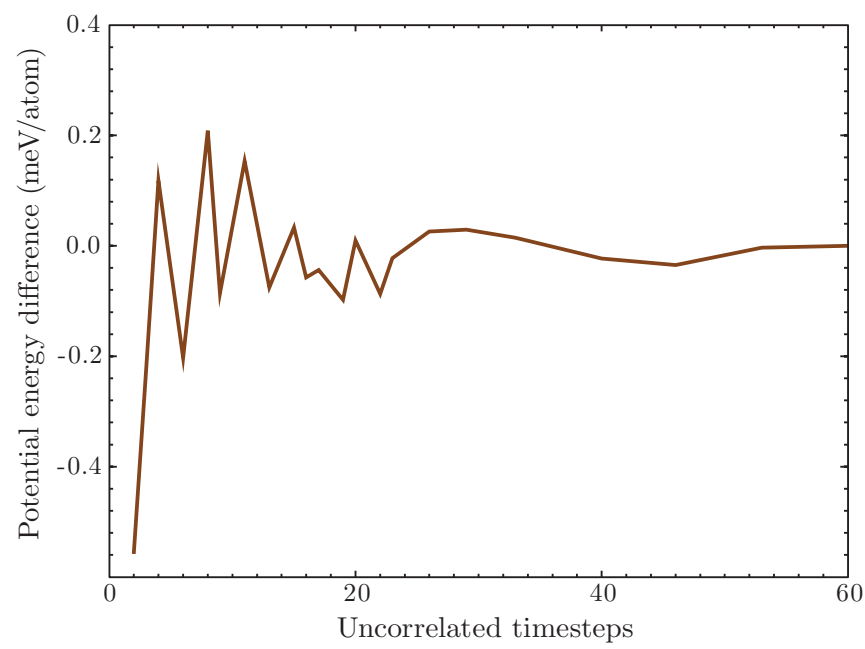

FIG. 2. (Color online) Convergence of the potential energy of our model potential for $\mathrm{Si}$. The displacements that go into Eq. (1) are from snapshots from molecular dynamics. 
volume change. They are given by

$$
\gamma_{\mathbf{q} s}=-\frac{V}{\omega_{\mathbf{q} s}} \frac{\partial \omega_{\mathbf{q} s}}{\partial V},
$$

where $V$ is the volume and $\omega_{\mathbf{q} s}$ is the frequency of mode $s$ at wave vector q. $\gamma_{\mathbf{q} s}$ can be obtained either by numerical differentiation of the phonon dispersion relations or from the third-order force constants: ${ }^{18,19}$

$$
\gamma_{\mathbf{q} s}=-\frac{1}{6 \omega_{\mathbf{q} s}^{2}} \sum_{i j k \alpha \beta \gamma} \frac{\epsilon_{i \alpha}^{\mathbf{q} s *} \epsilon_{j \beta}^{\mathbf{q} s}}{\sqrt{M_{i} M_{j}}} r_{k}^{\gamma} \Psi_{i j k}^{\alpha \beta \gamma} e^{i \mathbf{q} \cdot \mathbf{r}_{j}} .
$$

Here $\epsilon_{i \alpha}^{\mathbf{q} s}$ is a component $\alpha$ associated eigenvector $\epsilon$ for atom $i$. $M_{i}$ is the mass of atom $i$, and $\mathbf{r}_{i}$ is the vector locating its position. To confirm the accuracy and consistency of our third-order force constants, we calculated the mode Grüneisen parameters using both Eqs. (13) and (14), as can be seen in Fig. 3, the results are excellent, both in terms of consistency with each other and with respect to the experimental values.

With the third-order force constants we can calculate the phonon lifetimes. The lifetime due to phonon-phonon scattering is related to the imaginary part of the phonon self-energy ${ }^{20}$

$$
\frac{1}{\tau_{\mathbf{q} s}}=2 \Gamma_{\mathbf{q} s,}
$$

where $\tau_{\mathbf{q} s}$ is the lifetime for wave vector $\mathbf{q}$ and mode $s$, and

$$
\begin{aligned}
& \Gamma_{\mathbf{q} s}=\sum_{s^{\prime} s^{\prime \prime}} \frac{\hbar \pi}{16} \iint_{\mathrm{BZ}}\left|\Psi_{s s^{\prime} s^{\prime \prime}}^{\mathbf{q} \mathbf{q}^{\prime} \mathbf{q}^{\prime \prime}}\right|^{2} \Delta_{\mathbf{q q ^ { \prime }} \mathbf{q}^{\prime \prime}} \\
& \times\left[\left(n_{\mathbf{q}^{\prime} s^{\prime}}+n_{\mathbf{q}^{\prime \prime} s^{\prime \prime}}+1\right) \delta\left(\omega_{\mathbf{q} s}-\omega_{\mathbf{q}^{\prime} s^{\prime}}-\omega_{\mathbf{q}^{\prime \prime} s^{\prime \prime}}\right)\right. \\
& \left.+2\left(n_{\mathbf{q}^{\prime} s^{\prime}}-n_{\mathbf{q}^{\prime \prime} s^{\prime \prime}}\right) \delta\left(\omega_{\mathbf{q} s}-\omega_{\mathbf{q}^{\prime} s^{\prime}}+\omega_{\mathbf{q}^{\prime \prime} s^{\prime \prime}}\right)\right] d \mathbf{q}^{\prime} d \mathbf{q}^{\prime \prime} \text {. }
\end{aligned}
$$

$n_{\mathbf{q} s}$ is the equilibrium occupation number. The $\Delta_{\mathbf{q q} \mathbf{q}^{\prime \prime}}$ ensures momentum conversation $\mathbf{q}+\mathbf{q}^{\prime}+\mathbf{q}^{\prime \prime}=\mathbf{G}$, and the delta functions in frequency ensure energy conservation. The three-

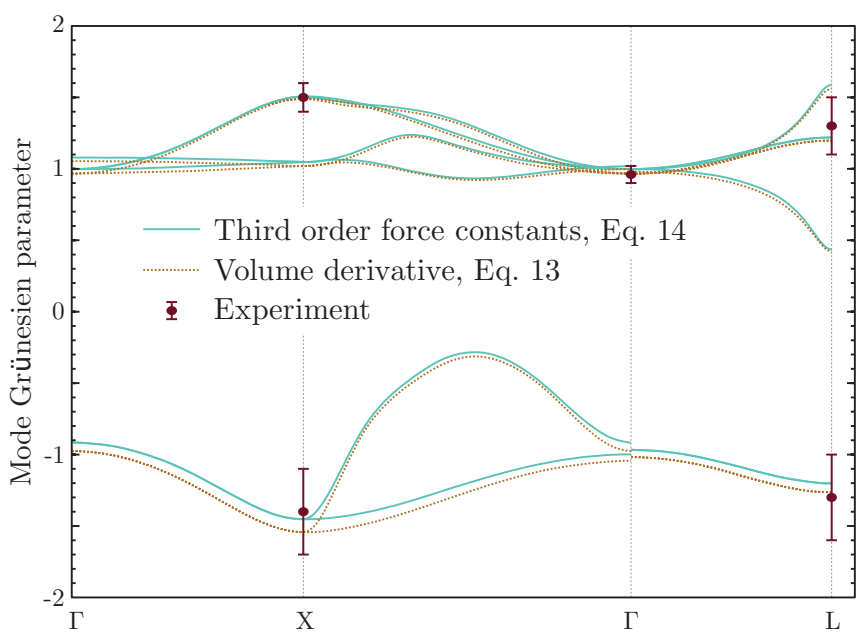

FIG. 3. (Color online) Mode Grüneisen parameters for Si. The solid line is calculated according to Eq. (14) and the dotted line according to Eq. (13). The experimental points are from Weinstein et al. (Ref. 17).

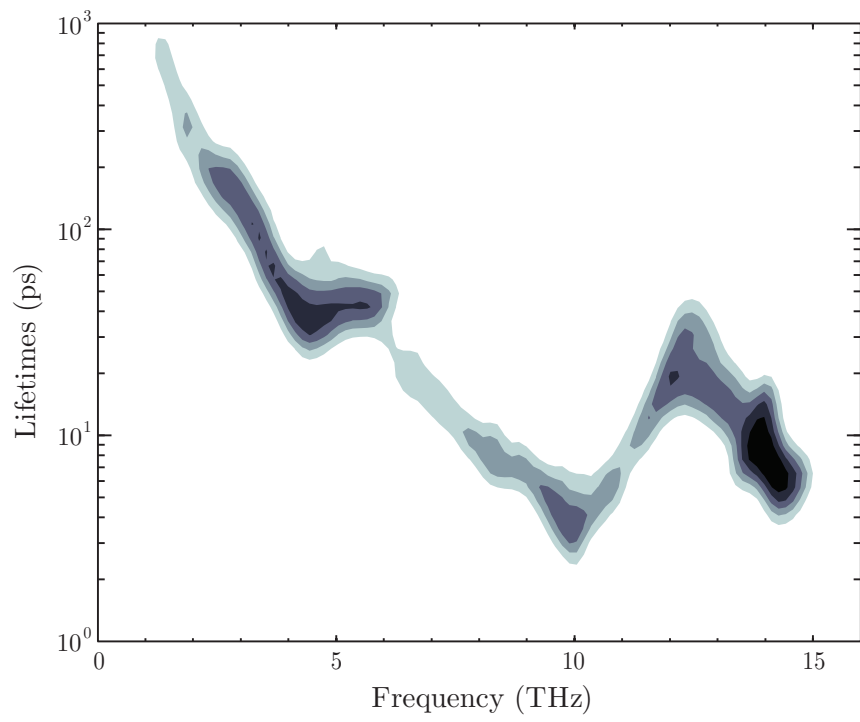

FIG. 4. (Color online) Density plot of phonon lifetimes in $\mathrm{Si}$ at $300 \mathrm{~K}$. The intensity is logarithmic.

phonon matrix elements are given by

$$
\begin{aligned}
\Psi_{s s^{\prime} s^{\prime \prime}}^{\mathbf{q} \mathbf{q}^{\prime \prime}}= & \sum_{i j k} \sum_{\alpha \beta \gamma} \frac{\epsilon_{\alpha i}^{\mathbf{q} s} \epsilon_{\beta j}^{\mathbf{q}^{\prime} s^{\prime}} \epsilon_{\gamma k}^{\mathbf{q}^{\prime \prime} s^{\prime \prime}}}{\sqrt{M_{i} M_{j} M_{j}} \sqrt{\omega_{\mathbf{q} s} \omega_{\mathbf{q}^{\prime} s^{\prime}} \omega_{\mathbf{q}^{\prime \prime} s^{\prime \prime}}}} \\
& \times \Psi_{i j k}^{\alpha \beta \gamma} e^{i \mathbf{q} \cdot \mathbf{r}_{i}+i \mathbf{q}^{\prime} \cdot \mathbf{r}_{j}+i \mathbf{q}^{\prime \prime} \cdot \mathbf{r}_{k}},
\end{aligned}
$$

where $M_{i}$ is the mass of atom $i, \epsilon_{\alpha i}^{\mathbf{q} s}$ is the component $\alpha$ of the eigenvector for mode $\mathbf{q} s$ and atom $i$, and $\mathbf{r}_{i}$ is the lattice vector associated with atom $i$. The resulting phonon lifetimes for $\mathrm{Si}$ can be seen in Fig. 4. The numerical integration is done on a $31 \times 31 \times 31$ Monkhorst-Pack ${ }^{21} q$-point grid. The momentum conservation is exactly fulfilled (the sum of two vectors on the grid ends up on the grid), and for the energy conservation we used the adaptive broadening scheme of Yates et al.. ${ }^{22}$ Our results agree well with previously calculated results. ${ }^{10,23}$

\section{TEMPERATURE DEPENDENCE}

The unique feature of our formalism if that the force constants are volume and temperature dependent. For comparison, only the volume dependence is included in the quasiharmonic approximation. In systems with dynamical instabilities, such as bcc $\mathrm{Zr}$, the temperature dependence is obvious, ${ }^{7}$ but it is also present in system that do not exhibit instabilities. $\epsilon$-FeSi is such a system. ${ }^{24}$

We ran Born-Oppenheimer molecular dynamics for $\mathrm{FeSi}$ out in a similar fashion to that of $\mathrm{Si}$, but we use a $3 \times 3 \times 3$ supercell (216 atoms), a cutoff of $300 \mathrm{eV}$, and used the $\Gamma$ point for Brillouin zone integration. We used the experimental lattice parameter of 4.779 and temperatures of 150 and $1200 \mathrm{~K}$ and ran the simulations for $30 \mathrm{ps}$ after equilibration with a 1 -fs time step. We used the same lattice constant at both temperatures to show that the softening is not due to thermal expansion, but originates from finite temperature effects, such as electronphonon coupling.

Phonon dispersion relations from TDEP calculations are shown in Fig. 5. We observe the softening across the whole spectrum with increasing temperature. The physical origin 


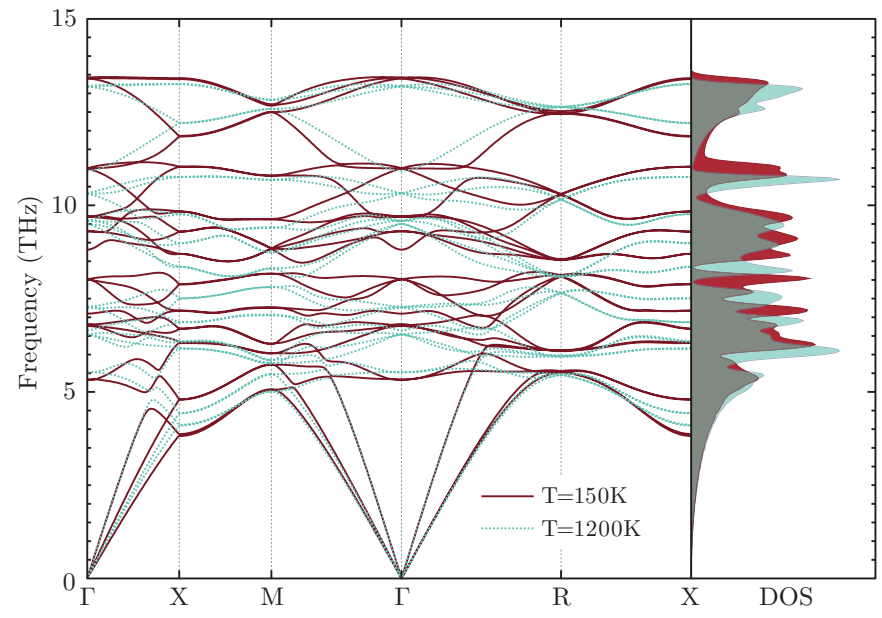

FIG. 5. (Color online) FeSi dispersion relations and phonon density of states. The solid lines correspond to $T=150 \mathrm{~K}$ and the dashed lines to $T=1200 \mathrm{~K}$. The experimentally observed softening across the spectrum can be seen clearly.

is discussed by Delaire et al. ${ }^{24}$ in great detail. It exhibits anomalous softening with temperature due to the thermal excitations smearing a sharp peak at the Fermi level, inducing a insulator-metal transition. We observe that TDEP can capture this temperature dependence well. The third-order force constants are also temperature dependent, the result of this can be seen in Fig. 6. The lifetimes are decreased significantly, across the whole spectrum. The temperature parameter used when evaluating lifetimes was fixed at $400 \mathrm{~K}$, the only difference comes from the temperature dependence of the force constants. This is consistent with experimental results, ${ }^{25}$ where they see a strong suppression of phonon linewidths below $250 \mathrm{~K}$. Due to the overestimation of the band gap in DFT, the closing of the gap occurs at a higher temperature in simulations [it is not fully closed until $1200 \mathrm{~K}$ (Ref. 24)], but the effect on the phonon linewidths and dispersions is qualitatively correct.

This tells us that to accurately describe the phonon-phonon interactions of $\mathrm{FeSi}$ at finite temperature, one needs to take the temperature dependence of the potential energy surface into account. A traditional approach where the force constants are calculated as derivatives of the potential energy at the ideal $T=0$ positions cannot describe this, even when extended to

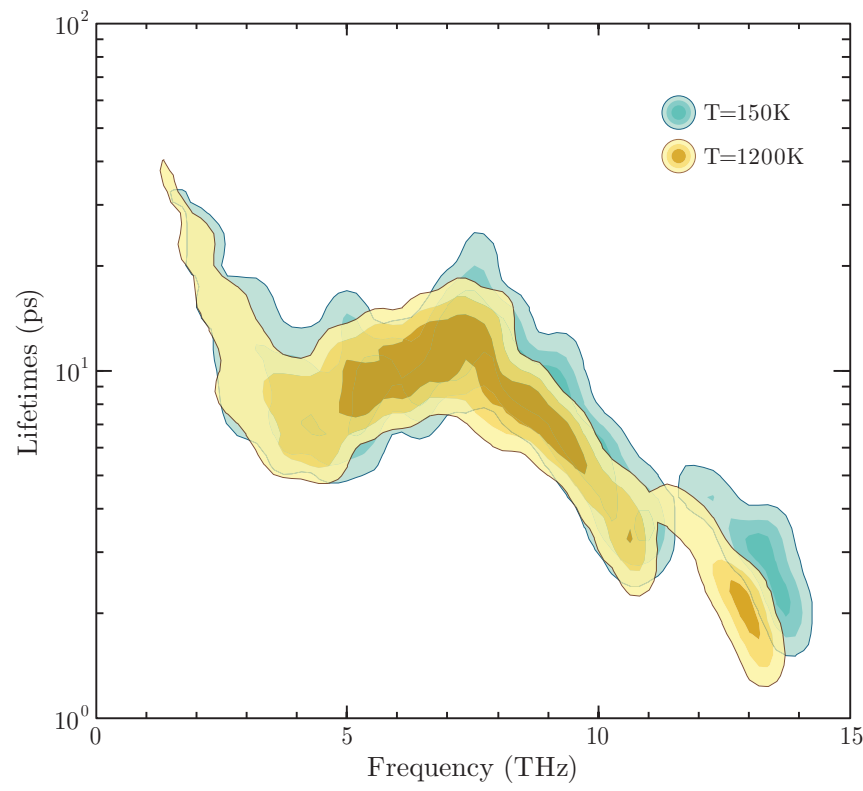

FIG. 6. (Color online) FeSi phonon lifetimes. The liftetimes are evaluated at $400 \mathrm{~K}$ using force constants extracted from 150 and $1200 \mathrm{~K}$.

orders beyond the third. For the insulator-metal transition to occur, the atoms need to occupy thermal excited positions.

\section{CONCLUSION}

We have presented an extension of our existing formalism to calculate temperature-dependent third-order force constants. They are shown to reproduce experimental results well. This is a numerically efficient technique to simultaneously incorporate all orders of phonon-phonon and electron phonon coupling into a model Hamiltonian.

\section{ACKNOWLEDGMENTS}

Support from the Knut and Alice Wallenberg Foundation (KAW) project "Isotopic Control for Ultimate Material Properties" and the Swedish Foundation for Strategic Research (SSF) program SRL10-002 is gratefully acknowledged. Supercomputer resources were provided by the Swedish National Infrastructure for Computing (SNIC).
${ }^{1}$ M. Born and K. Huang, Dynamical Theory of Crystal Lattices (Oxford University Press, Oxford, 1964) .

${ }^{2}$ A. A. Maradudin and S. Vosko, Rev. Mod. Phys. 40, 1 (1968).

${ }^{3}$ G. P. Srivastava, The Physics of Phonons (A. Hilger, Bristol, England, 1990)

${ }^{4}$ D. A. Broido, M. Malorny, G. Birner, N. Mingo, and D. A. Stewart, Appl. Phys. Lett. 91, 231922 (2007).

${ }^{5}$ L. Lindsay, D. A. Broido, and T. L. Reinecke, Phys. Rev. Lett. 109, 095901 (2012).

${ }^{6}$ S. Narasimhan and D. Vanderbilt, Phys. Rev. B 43, 4541 (1991).
${ }^{7}$ O. Hellman, I. A. Abrikosov, and S. I. Simak, Phys. Rev. B 84, 180301 (2011).

${ }^{8}$ O. Hellman, P. Steneteg, I. A. Abrikosov, and S. I. Simak, Phys. Rev. B 87, 104111 (2013).

${ }^{9}$ M. Omini and A. Sparavigna, Nuovo Cimento Soc. Ital. Fis. D 19, 1537 (1997).

${ }^{10}$ J. A. Pascual-Gutierrez, J. Y. Murthy, and R. Viskanta, J. Appl. Phys. 106, 063532 (2009).

${ }^{11}$ G. Kresse and D. Joubert, Phys. Rev. B 59, 1758 (1999).

${ }^{12}$ G. Kresse and J. Furthmüller, Phys. Rev. B 54, 11169 (1996).

${ }^{13}$ G. Kresse and J. Hafner, Phys. Rev. B 48, 13115 (1993). 
${ }^{14}$ G. Kresse, Comput. Mater. Sci. 6, 15 (1996).

${ }^{15}$ S. Nosé, Mol. Phys. 52, 255 (1984).

${ }^{16}$ J. P. Perdew, K. Burke, and M. Ernzerhof, Phys. Rev. Lett. 77, 3865 (1996).

${ }^{17}$ B. Weinstein and G. Piermarini, Phys. Rev. B 12, 1172 (1975).

${ }^{18}$ J. Fabian and P. B. Allen, Phys. Rev. Lett. 79, 1885 (1997).

${ }^{19}$ D. A. Broido, A. Ward, and N. Mingo, Phys. Rev. B 72, 014308 (2005).

${ }^{20}$ D. C. Wallace, Thermodynamics of Crystals, Dover Books on Physics (Dover, New York, 1998) .
${ }^{21}$ H. Monkhorst and J. Pack, Phys. Rev. B 13, 5188 (1976).

${ }^{22}$ J. R. Yates, X. Wang, D. Vanderbilt, and I. Souza, Phys. Rev. B 75, 195121 (2007).

${ }^{23}$ K. Esfarjani, G. Chen, and H. Stokes, Phys. Rev. B 84, 085204 (2011).

${ }^{24}$ O. Delaire, K. Marty, M. B. Stone, P. R. C. Kent, M. S. Lucas, D. L. Abernathy, D. Mandrus, and B. C. Sales, Proc. Natl. Acad. Sci. 108, 4725 (2011).

${ }^{25}$ P. Nyhus, S. L. Cooper, and Z. Fisk, Phys. Rev. B 51, 15626 (1995). 